\title{
Effect of Fusarium Mycotoxin Zearalenone on Gut Epithelium
}

\author{
Ionelia TARANU*1), Aurora ARGHIR ${ }^{2)}$, Gina PISTOL ${ }^{1)}$, Daniela MARIN ${ }^{1)}$, Ana-Maria NICULESCU³ \\ ${ }^{1)}$ National Institute for Research and Development for Biology and Animal Nutrition, 1 Bucuresti Road, \\ 077015 Balotesti, Ilfov, Romania \\ ${ }^{2)}$ National Institute of Pathology Victor Babes, 99-101 Independentei Road, Bucharest, Romania \\ ${ }^{3}$ Agilrom Scientific,. 1H Pipera Tunari Road, Bucharest, Romania \\ *Corresponding author, email: ionelia.taranu@ibna.ro \\ Bulletin UASVM Animal Science and Biotechnologies 71(2) / 2014, \\ Print ISSN 1843-5262; Electronic ISSN 1843-536X \\ DOI: $10.15835 /$ buasvmcn-asb:10543
}

\begin{abstract}
In the present study, in vitro experiments were conducted to evaluate the changes produced by fusariotoxin, Zearalenone (ZEA), on global gene expression in human intestinal epithelial cells (Caco2), knowing that the intestine is the first organ exposed and affected by the mycotoxin ingestion during an oral intoxication. Caco2 cells were treated with $10 \mu \mathrm{M}$ of ZEA for 24 hours and the changes in global genes expression was investigated by microarray and analysed by using Gene Spring GX v.11.5 software. Our results showed that ZEA at the concentration of $10 \mu \mathrm{M}$ did not affect the cell viability but, the microarray analyses revealed that this mycotoxin was able to induced significant changes in genes expression profile in Caco2 cells in comparison with control cells. The upregulation gene expression was the most common effect produced by ZEA in Caco 2 cells after 24h of exposure to $10 \mu \mathrm{M}$ of ZEA. Microarray analyses identified 377 genes differentially regulated of which 11 genes were downregulated and 319 up-regulated with a fold change $\geq 2$. ZEA was also able to increase the expression of certain cytokines (IL-1 $\beta$, IL-6, IL-8 and IFN- $\gamma$ ) involved in inflammation, signalling molecules (ERK1, ERK2, JNK1, JNK2, p38- $\alpha$ ) and nuclear receptors (NF-kB and PPAR- $\gamma$ ), which control the immune functions. Together all these results suggest that zearalenone is a genotoxic mycotoxin able to induced alterations in a significant number of genes involved in different molecular and functional processes at intestinal level.
\end{abstract}

Keywords: zearalenone, gene expression, microarray, human intestinal Caco-2 cells

Introduction. Zearalenone is a mycotoxin produced by Fusarium sp, very known for its oestrogenic properties (Tabuc et al., 2009). In addition, ZEA has been shown to be immunotoxic and hepatonephrotoxic, which means a greater risk factor for public health (Tiemann and Dänicke, 2007; Kuiper et al., 1998). Although the studies on ZEA intensified in the last period, the mechanisms of its toxicity need to be evaluated. Considering that the intestine represents the main route of intoxication with mycotoxins (Pinton et al., 2010) a microarray approach could generate important genomic overview necessary to elucidate the interaction of this toxin with the genes involved in different metabolic processes (proliferation and cell differentiation, apoptosis, inflammation, protein synthesis etc) at intestinal level.

Aims and objectives. In the present study, in vitro experiments were conducted to evaluate the changes produced by ZEA on global gene expression of epithelial intestinal cells taking into consideration that the intestine is the first organ exposed and affected by the mycotoxin ingestion during an oral intoxication.

Materials and methods. Caco- 2 cells were seeded at $2.0 \times 10^{5}$ cells concentration in 24well cell culture plates (Costar, Corning, NY, USA) until they reached confluence (2-3 days). Cells were treated were with $10 \mu \mathrm{M}$ of pure ZEA dissolved in ethanol/culture media (1:1, v:v) for 


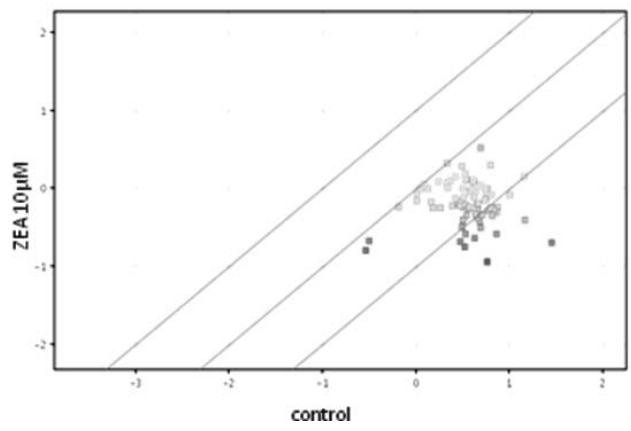

Fig. 1a. Microarray down-regulated genes.

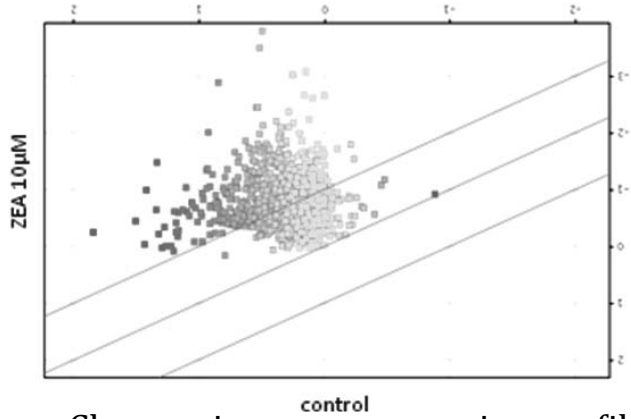

24 hours. Changes in genes expression profile was

Fig. 1b. Microarray up-regulated genes

investigated by microarray and qPCR using Agilent technology and methodology. Agilent Gene Spring GX v.11.5 software was used to analyze expression profile of altered genes. Genes with fold change $\geq 2$ and a $P$ value $<0.05$ were considered differentially expressed.

Results and discussion. Our results showed that ZEA at the concentration of $10 \mu \mathrm{M}$ did not affect the cell viability but the microarray analyses revealed that this mycotoxin was able to induced significant changes in genes expression profile in Caco 2 cells in comparison with control cells. The up-regulation gene expression was the most common effect produced by ZEA in Caco 2 cells after $24 \mathrm{~h}$ of exposure to $10 \mu \mathrm{M}$ of ZEA. Thus, Microarray analyses identified 377 genes differentially regulated of which 11 genes were down-regulated and 319 up-regulated with a fold change $\geq 2$ (Fig.1a and Fig.1b). 102 of upregulated genes had a fold change between 3 and 6 . The highest expressed gene (19.26 fold change) was STRN3 gene encoding for calmodulin protein, which mediate many crucial processes such as inflammation, metabolism, apoptosis etc.

ZEA was also able to increase the expression of other marker of inflammation like cytokines (IL-1 $\beta$, IL-6, IL-8 and IFN- $\gamma$ ), signaling molecules (ERK1, ERK2, JNK1, JNK2, p38- $\alpha$ ) and nuclear receptors (NF-kB and PPAR- $\gamma$ ), which control the immune functions.

Conclusion. Together all these results suggest that zearalenone is a mycotoxin, which produce genotoxic effect able to induced alterations in a significant number of genes involved in different molecular and functional processes at intestinal level.

Acknowledgements. This work was supported by funds from the National Research Project PNIIID PCE 2011-3-0339-101/2011-2015 granted by the Romanian Ministry of Research and Technology.

\section{REFERENCES}

1. Tabuc C, Marin D, Guerre P, Sesan T, Bailly JD (2009). Molds and mycotoxin content of cereals in southeastern Romania. J Food Prot 72: 662-665.

2. Tiemann U, Dänicke S (2007). In vivo and in vitro effects of the mycotoxins zearalenone and deoxynivalenol on different non-reproductive and reproductive organs in female pigs: a review. Food Addit Contam 24:306-314.

3. Kuiper GGJM, Lemmen JG, Carlsson B, Corton JC, Safe SH, Van der Saag PT, Van der Burg B, Gustafsson JA (1998). Interaction of estrogenic chemicals and phytoestrogens with estrogen receptor b. Endocrinology 139:4252-4263.

4. Pinton P, Braicu C, Nougayrède J-P, Laffitte, J, Taranu I and Oswald IP (2010). Deoxynivalenol impairs porcine intestinal barrier function and decreases the protein expression of claudin-4 through a mitogen-activated protein kinase-dependent mechanism. J. Nutr. 140: 19561962. 